\title{
INVO Procedure: Minimally Invasive IVF as an Alternative Treatment Option for Infertile Couples
}

\author{
Elkin Lucena, Angela M. Saa, Doris E. Navarro, \\ Carlos Pulido, Oscar Lombana, and Abby Moran \\ Centro Colombiano de Fertilidad y Esterilidad (CECOLFES) S.A.S., Calle 102 No. 14A-15, 56769 Bogotá, Colombia \\ Correspondence should be addressed to Elkin Lucena, cecolfes@cecolfes.com
}

Received 15 October 2011; Accepted 8 December 2011

Academic Editor: João B. A. Oliveira

Copyright (C) 2012 Elkin Lucena et al. This is an open access article distributed under the Creative Commons Attribution License, which permits unrestricted use, distribution, and reproduction in any medium, provided the original work is properly cited.

\begin{abstract}
Intravaginal culture (IVC), also called INVO (intravaginal culture of oocytes), is an assisted reproduction procedure where oocyte fertilization and early embryo development are carried out within a gas permeable air-free plastic device, placed into the maternal vaginal cavity for incubation. In the present study we assessed the outcome of the INVO procedure, using the recently designed INVOcell device, in combination with a mild ovarian stimulation protocol. A total of 125 cycles were performed. On average 6.5 oocytes per cycle were retrieved, and a mean of 4.2 were placed per INVOcell device. The cleavage rate obtained after the INVO culture was $63 \%$. The procedure yielded $40 \%, 31.2 \%$, and $24 \%$ of clinical pregnancy, live birth, and single live birth rates per cycle, respectively. Our results suggest that the INVO procedure is an effective alternative treatment option in assisted reproduction that shows comparable results to those reported for existing IVF techniques.
\end{abstract}

\section{Introduction}

Conventional in vitro fertilization (IVF) is the original technique of the so-called test-tube babies and currently an established treatment for infertility. The fertilization of the oocytes with the spermatozoa is performed in the laboratory, by simulating the physiological conditions to which the gametes are exposed in vivo. Intravaginal culture (IVC), also called INVO (intravaginal culture of oocytes), is a procedure developed by Ranoux et al., 1988, proposed as a simplified alternative option to conventional IVF [1]. In the procedure oocyte fertilization and early embryo development are carried out within a gas permeable $\left(\mathrm{CO}_{2}\right.$ and $\mathrm{O}_{2}$ ) air-free plastic device, placed into the maternal vaginal cavity for incubation $[1,2]$, thus replacing the complex IVF laboratory [3]. Few years ago, INVOcell device was specially designed to overcome the difficulties and improve the results obtained with the early prototype device, which explained the occurrence of a low diffusion of the procedure for years. This new device has been ISO 10993 tested (and mouse embryos tested) to assess toxicity and biocompatibility and has received the European Union CE mark declaration of conformity [3], which is equivalent to approval by the Food and Drug Administration (FDA) in the U.S.

INVO procedure has been used worldwide and results from cycles performed by infertility centers around the world, in countries such as France, Germany, The Netherlands, England, USA, and Japan, have been published [1, 2, 4-12]. More recently our center has pioneered the use of this procedure employing the INVOcell device within the Latin-American region [13] and has been leader in its recent introduction in countries such as Mexico, Guatemala, El Salvador, Nicaragua, Dominican Republic, Panama, Venezuela, Ecuador, Peru, Bolivia, and Brazil.

In this study, we employed the INVO procedure using the INVOcell device [14], in combination with a mild ovarian stimulation protocol, with the aim of evaluating its usefulness as an alternative treatment option for infertile couples, in terms of embryonary development, clinical pregnancy, and live birth rates.

\section{Materials and Methods}

2.1. Setting and Design. The present study was carried out at the Colombian Center for Fertility and Sterility 


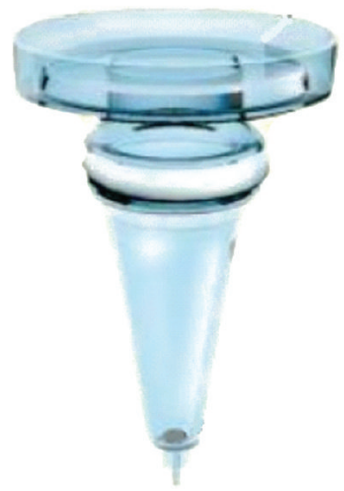

(a)

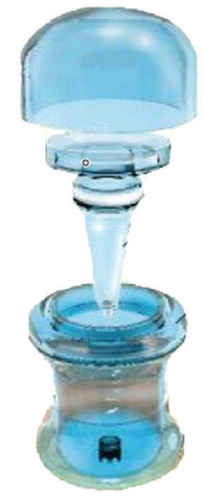

(b)

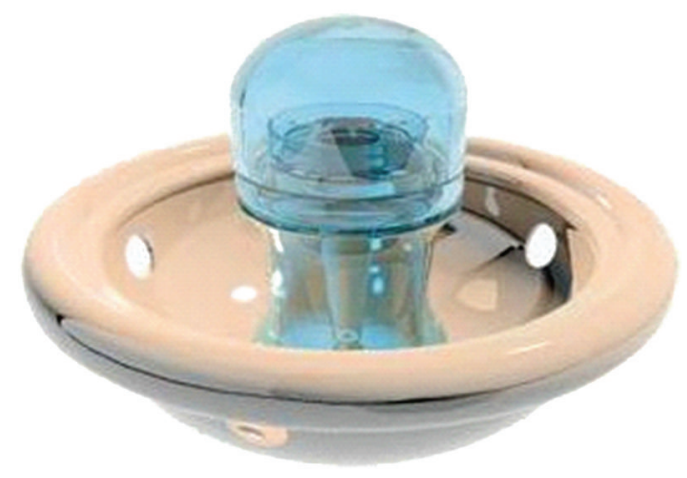

(c)

Figure 1: Component parts of the INVOcell device. (a) Inner chamber. (b) Outer rigid shell. (c) Assembled INVOcell device with its retention system.

(CECOLFES), Bogota, Colombia. The center is certified according to ISO 9001 version 2008, and the study was approved by the institutional ethics committee, which allowed retrospective review of the patients' files.

2.2. Study Population. One hundred twenty (120) infertile couples with over 2 years of infertility were admitted upon giving them information and receiving their informed consent for treatment using the INVO procedure. Exclusion criteria included severe endometriosis, polycystic ovary syndrome, egg donation, and severe male factor.

From June, 2009, through May, 2011, one hundred twenty five (125) INVO cycles were performed. Population was ranked by age among four different groups to report clinical pregnancy, live birth, and single live birth rates in order to compare the outcome of the INVO procedure. Patients were distributed in groups as follows: $\leq 29$, between 30 and 34, between 35 and 39, and $\geq 40$ years old.

2.3. Controlled Ovarian Stimulation. Mild ovarian stimulation protocol was started with ovarian quiescence using oral contraceptive pills containing $150 \mu \mathrm{g}$ of desogestrel and $30 \mu \mathrm{g}$ of ethinylestradiol (Marvelon Schering Plough., Bogota, Colombia) for 3 weeks. Clomiphene citrate (50 or $100 \mathrm{mg}$; Omifin Cipla Ltd., Bernagoa, India) and human menopausal gonadotropin (one or two ampoules of $75 \mathrm{IU}$; Merional IBSA, Switzerland) were administered starting on day three of the following cycle until follicles reached $14-16 \mathrm{~mm}$ in diameter; at that point, blockage of follicular rupture to prevent spontaneous ovulation was achieved by administration of Indomethacin ( $50 \mathrm{mg}$; Genfar, Cali, Colombia) three times per day until oocyte retrieval [15]. When the dominant follicle(s) reached $17-18 \mathrm{~mm}$ in diameter human chorionic gonadotropin (10,000 IU; Gonacor Massone, Buenos Aires, Argentina) was injected, and 36 hours later transvaginal oocyte retrieval was performed under ultrasound guidance by traditional techniques [16].
2.4. Oocytes Selection. The maturity of the oocytes was morphologically assessed as traditionally, under stereo microscope with 8x magnification, based upon the expansion and radiance of the cumulus/corona oocyte complex (COC) [17], using criteria such as cumulus viscosity and dissociation of the cumulus corona radiate, additionally when possible nuclear maturity was confirmed by observation of the polar body.

2.5. Intravaginal Culture Procedure. The INVOcell device (INVO Bioscience., Beverly, MA, USA, http://www.invobioscience.com/) is composed of an inner chamber with a rotating valve and a protective outer rigid shell (Figure 1). The INVO procedure was performed as previously described with some modifications [3]. Semen samples were treated by the swim-up method [18]. Initially the device inner chamber is filled with pregazed and prewarmed G2 Plus version 5 medium (Vitrolife AB, Goteborg, Sweden), then a count of 35.000-50.000 spermatozoa are loaded, followed by the selected number of oocytes (4.2 in average). After assembly of the device, it is immediately positioned into the vaginal cavity, in proximity to the uterine cervix, altogether with a diaphragm as retention system. Some recommendations were given to patients to follow during the culture period such as restraining from intercourse and abstention of tub bathing, douching, or swimming.

After a 72-hour culture period the INVOcell device was removed, embryos were retrieved and immediately evaluated according to their development and fragmentation degree. The selected embryos were transferred under transabdominal ultrasound guidance.

2.6. Luteal Phase Support and Pregnancy Determination. Estradiol Valerianate ( $4 \mathrm{mg}$; Delpharm Lille SAS, Paris, France) and natural oily progesterone (100 mg; Ryan Laboratories, Bogota, Colombia) were given daily during 6 days starting the day of transfer, followed by vaginal progesterone (600 mg/day; Utrogestan Besins International, Paris, France) 
TABLE 1: Summary of results from the INVO procedure.

\begin{tabular}{lccccccc}
\hline Groups $^{\mathrm{a}}$ & Cycles $(n)$ & Transfers $^{\mathrm{b}}(n)$ & Retrieval $^{\mathrm{c}}$ & INVOcell $^{\mathrm{d}}$ & Cleavage $^{\mathrm{e}}$ & ET $^{\mathrm{f}}$ & Pregnancy $^{\mathrm{g}}$ \\
\hline$\leq 29$ & 17 & $16(94,1)$ & 7,53 & 4,59 & $2,7(58,7)$ & 2.3 & $10(58,8)$ \\
$30-34$ & 54 & $51(94,4)$ & 6,52 & 3,8 & $2,8(73,7)$ & 2.3 & $22(40,7)$ \\
$35-39$ & 48 & $43(89,6)$ & 6,23 & 4,27 & $2,5(58,5)$ & 2.0 & $17(35,4)$ \\
$\geq 40$ & 6 & $4(66,7)$ & 5,5 & 5 & $1,5(30)$ & 1.5 & $1(16,66)$ \\
\hline Total & 125 & $114(91,2)$ & 6,5 & 4,2 & $2,6(63)$ & 2,1 & $50(40)$ \\
\hline
\end{tabular}

INVO: intravaginal culture of oocytes; ET: embryo transfer. Values in parentheses are percentages.

${ }^{a}$ Ranges of ages. ${ }^{b}$ Cycles that ended up in transfer. ${ }^{c}$ Mean number of retrieved oocytes per punction. ${ }^{\mathrm{d}}$ Mean number of oocytes placed for fertilization per INVOcell device (cycle). ${ }^{\mathrm{e}}$ Mean number of retrieved viable proper developed embryos per INVOcell device (cycle). ${ }^{\mathrm{f}}$ Mean number of transferred embryos per cycle. ${ }^{\mathrm{g}}$ Number of clinical pregnancies per cycle.

until day 12 after-transfer, when serum $\beta$-HCG pregnancy determination was performed. Seven weeks after transfer, the presence of gestational sac with fetal heart beat by ultrasonography was used to confirm the clinical pregnancy.

2.7. Vitrification. In some patients, supernumerary oocytes confirmed to be mature by first polar body observation after denudation, as well as those good quality embryos that were not transferred, were vitrified for use in subsequent cycles. Vitrification was performed according to the protocol previously published by our group [19].

2.8. Outcome Measures and Statistical Analysis. Primary outcome measures included pregnancy, live birth, and single live birth rates per transfer. Secondary outcome measures included mean numbers of retrieved oocytes and oocytes placed per INVO device, as well as embryo cleavage and transfer rates after INVO culture. Statistical analysis, to compare our results with published data concerning conventional IVF outcomes, was performed using Student's $t$-test. $P<$ 0.05 was considered statistically significant.

\section{Results}

One hundred twenty-five (125) cycles combining the INVO procedure and mild ovarian stimulation protocol were performed. There was no cycle cancellation, as all started cycles went to retrieval. A total of 812 oocytes were retrieved, for an average of 6.5 per puncture. A mean of 4.2 oocytes were placed for insemination per the INVO device. On average 2.6 embryos per cycle were obtained, for a cleavage rate of $63 \%$, out of which a mean of 2.1 embryos were transferred per cycle, for a total of 114 transfers (91.2\%). Cycle distribution per patient's age groups was seventeen for $\leq 29,54$ for $30-34,48$ for $35-39$, and 6 for $\geq 40$ years old (Table 1).

3.1. Patients $\leq 29$ Years Old. A total of 128 oocytes were retrieved (mean value 7.5 ), out of which 78 were selected for insemination (average 4.6). After intravaginal culture, 46 embryos (on average 2.7 per cycle) were obtained, out of which an average of 2.3 embryos per cycle were selected for transference, and the remaining viable embryos were cryopreserved. Twelve positive $\beta$-HCG tests were obtained, out of which 10 clinical pregnancies were confirmed (Table 1). Up to date 6 pregnancies have successfully reached live birth with eight healthy children born, and there is one ongoing pregnancy.

3.2. Patients between 30 and 34 Years Old. A total of 352 oocytes were retrieved (mean value 6.5), out of which 207 were selected for insemination (average 3.8). After intravaginal culture, 152 embryos (on average 2.8 per cycle) were obtained, out of which an average of 2.3 embryos per cycle were selected for transference, and the remaining viable embryos were cryopreserved. Twenty-six positive $\beta$-HCG tests were obtained, out of which 22 clinical pregnancies were confirmed (Table 1 ). Up to date 12 pregnancies have successfully reached live birth and 17 healthy children were born, and there are 5 ongoing pregnancies.

3.3. Patients between 35 and 39 Years Old. A total of 229 oocytes were retrieved (mean value 6.2), out of which 205 were selected for insemination (average 4.3). After intravaginal culture, 121 embryos (on average 2.5 per cycle) were obtained, out of which an average of 2.0 embryos per cycle were selected for transference, and the remaining viable embryos were cryopreserved. Eighteen positive $\beta$-HCG tests were obtained, out of which 17 clinical pregnancies were confirmed (Table 1). Up to date 12 pregnancies have successfully reached live birth and 17 healthy children were born, and there are 2 ongoing pregnancies.

3.4. Patients $\geq 40$ Years Old. A total of 33 oocytes were retrieved (mean value 5.5), out of which 30 were selected for insemination (average 5.0). After intravaginal culture, 9 embryos (on average 1.5 per cycle) were obtained, out of which an average of 1.5 embryos per cycle were selected for transference, and the remaining viable embryos were cryopreserved. One clinical pregnancy was confirmed (Table 1), up to date this is a healthy ongoing pregnancy.

Out of the 125 cycles, 11 were not transferred and in two of them there were well-developed embryos but transfer could not be achieved, one because an abnormal uterine bleeding and the other due to the presence of bicornuate uterus in retroversion, in both cases embryos were vitrified. In the remaining 9 cycles there was either not fertilization (seven) or an inappropriate embryo development (two). 


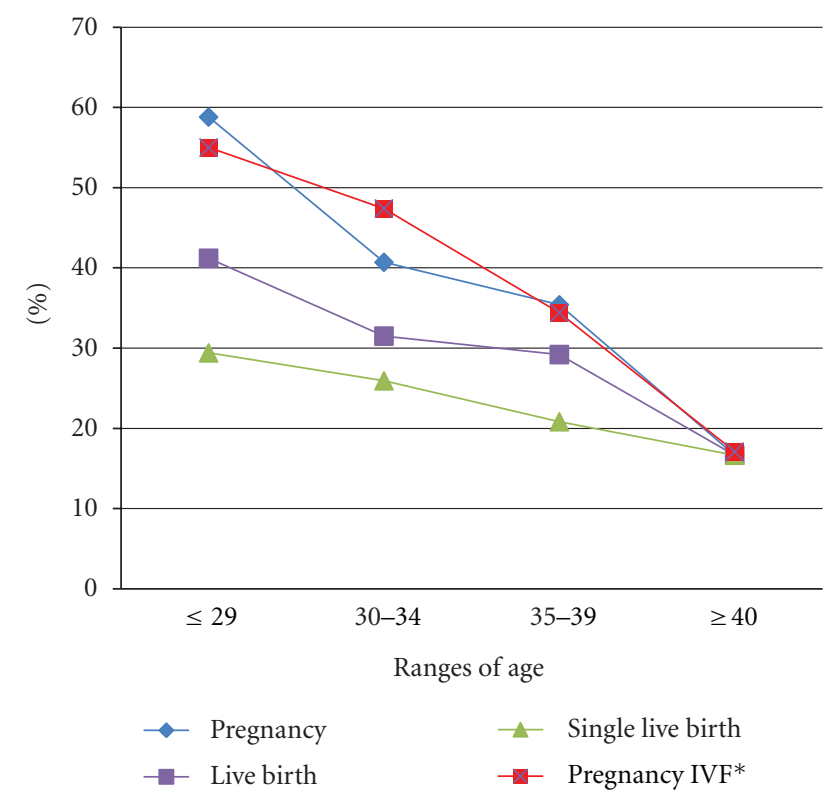

FIGURE 2: Rates of primary outcomes: pregnancy, live birth, and singleton live birth from INVO procedure. ${ }^{*}$ From the US Centers for Disease Control and Prevention (CDC), 2009 National Summary Report. $P>0.05$ for pregnancy rates between traditional IVF versus INVO ( $T$-test). For INVO results, live birth and single live birth rates include up to date ongoing pregnancies.

None of the 120 patients reported any discomfort while using the INVOcell device or presented signs of infection.

\section{Discussion}

In the present study we assessed the outcome of the INVO procedure, using the specially designed INVOcell device, in terms of pregnancy, live birth, and single live birth rates; our results showed comparable successful rates with traditional IVF, highlighting its usefulness as an alternative option treatment in assisted reproduction.

The US Centers for Disease Control and Prevention (CDC) provides cumulative summary statistics on the outcomes of IVF cycles; the most recent complete data available are from 2008 [20]. During that year the pregnancy, live birth, and singleton live birth rates per oocyte retrieval were $41.6 \%, 33.8 \%$, and $23 \%$, respectively. These results are comparable to ours from INVO cycles as we obtained $40 \%, 31.2 \%$, and 24\%, respectively, for these outcomes. Patients' age was the most predictive factor of success with a marked decrease across the groups of age from $\leq 29$ until $\geq 40$ years old, in terms of pregnancy, live birth, and single live birth rates (Figure 2). More recently available data report a pregnancy rate per cycle of 55\% for patients of 29 years old and under, $47 \%$ for patients between 30 and $34,34 \%$ for patients between 35 and 39 , and $17 \%$ for patients 40 years old and older [21]. These results are also comparable to ours for the INVO procedure, where as expected pregnancy rates decreased with maternal age, from $58.8 \%$, through $40.7 \%$ and $35.4 \%$, until $16.7 \%$, for woman aged $\leq 30,30-34$, 3439 , and $\geq 40$ years old, respectively. There were no statistical differences for pregnancy rate through age groups between the reported data and our results $(P=0.96)$ (Figure 2). Taken together, these results suggest that the INVO procedure could be an alternative treatment for infertile patients ensuring success rates comparable to those in the existing IVF techniques.

Cycle secondary outcomes, including mean numbers of retrieved oocytes and oocytes placed per INVO device, in addition to embryos cleavage and transfer rates after INVO culture, were comparable between the first three established groups of age. However, the last group of age ( $\geq 40$ years old) showed notably lower mean values within these measures (Table 1).

The higher incidence of multiple pregnancies secondary to IVF is well recognized. The $2008 \mathrm{CDC}$ annual report indicates a $55.5 \%$ of singleton and $26 \%$ of multiple live births (20). In comparison, in our results the multiple gestation rate was 1.4 times less (18\%) than the reported. Our pregnancies were distributed as follows: $60 \%$ single, $12 \%$ twins, and $6 \%$ triple live births, as a result of transferring on average 2.4 embryos per transferred cycle. The advances of ART have involved among other things evolution of culture media components; however, the environmental conditions influence drastically the embryo quality by their effect on $\mathrm{O}_{2}$ and $\mathrm{CO}_{2}$ concentration, $\mathrm{pH}$, and temperature [22-25]; a misbalance in this components may induce oxidative stress, responsible for inappropriate early development and embryo fragmentation [26]. Oxygen is an important player during embryo development [27]; the INVO procedure offers an in vivo fertilization environment with the effectiveness of an oxygen concentration that closely resembles the uterine cavity atmosphere of less than $5 \%$ of oxygen. This concentration of oxygen ensures the energetic metabolism required for a successful gametes viability, activation, fertilization, and embryo development, which takes place under near anaerobic conditions [28]. On the other hand, $\mathrm{CO}_{2}$ is a key atmospheric component during embryo development; in the culture media it produces carbonic acid that is equilibrated with sodium bicarbonate originating the optimal $\mathrm{pH}$ necessary for embryo development [29]. In traditional large gas-filled incubators, the $\mathrm{CO}_{2}$ concentrations may vary because of repeated opening, impacting the $\mathrm{pH}$ equilibrium in the culture medium, decreasing embryo quality [30]. In contrast the INVOcell device is a closed system able to keep temperature and $\mathrm{pH}$ stability during $72 \mathrm{~h}$ of uninterrupted embryo culture, providing a stable, pure, inexpensive, and easy-access source of the required oxygen/ $/ \mathrm{CO}_{2}$ concentration. A limitation of the intravaginal culture would be the inability to monitor or adjust gas levels; however, according to our results we could ensure that this system is capable of maintaining an environmental stability that achieves comparable results to those obtained with large gas-filled incubators $[29,31]$. In our study, from 520 oocytes loaded into the INVOcell device, 326 viable embryos (with a proper development of 6-8 cells after 72 hours of culture) were retrieved; additionally $79.6 \%$ of these embryos were free of fragmentation. These results suggest that the INVOcell 
procedure offers an appropriate embryo development with an optimal quality for implantation success. Infertile couples during assisted reproduction treatments undergo an intense process that involves physiological difficulties, physical discomfort, psychological and economical implications, in addition to the medical procedures that represent risks and secondary effects $[32,33]$. A significant aspect of the INVO procedure lies in the psychological benefit that is created among the patients who feel closely involved in the process of fertilization and early embryo development, what generates a high level of acceptance of the procedure. The INVOcell is placed into the vaginal cavity altogether with a retention system (diaphragm) (Figure 1(c)) that situates it close to the uterus, the holes in the diaphragm membrane avoid the accumulation of vaginal secretions that would be responsible for infection induction during intravaginal culture period. Accordingly in our study no patient reported any infection or physical discomfort while or after using the device.

An advantage of the INVO procedure is the fact that by simplifying the laboratory equipment and manipulation needed, it might decrease the costs and allow a widespread application for patients who cannot afford traditional IVF [34], as it would be the case in developing countries, where the access to cost-effective infertility treatments is limited.

This study suggests that intravaginal culture using the INVOcell device could be a viable alternative option for assisted reproduction. However, additional prospective and probably multicentric studies, involving higher number of cycles, would be necessary to confirm its efficacy and safety. Additionally it would be interesting to investigate the outcome and usefulness of the INVO culture system in conjunction with the ICSI technique, to evaluate its applicability for indications such as severe male factor.

\section{Acknowledgments}

The authors would like to express their appreciation to Clara Esteban, Ph.D., and Eliana Bonilla M.S., for the editing and critical review of this paper. They apologize to those whom they have not cited owing to space limitation.

\section{References}

[1] C. Ranoux, F. X. Aubriot, J. B. Dubuisson et al., "A new in vitro fertilization technique: intravaginal culture," Fertility and Sterility, vol. 49, no. 4, pp. 654-657, 1988.

[2] M. Fukuda, K. Fukuda, and C. Ranoux, "Unexpected low oxygen tension of intravaginal culture," Human Reproduction, vol. 11, no. 6, pp. 1293-1295, 1996.

[3] R. Frydman and C. Ranoux, "INVO: a simple, low cost effective assisted reproductive technology," ESHRE Monographs, vol. 1, Article ID den163, pp. 85-89, 2008.

[4] K. Sterzik, B. Rosenbusch, V. Sasse, A. Wolf, H. M. Beier, and C. Lauritzen, "A new variation of in-vitro fertilization: intravaginal culture of human oocytes and cleavage stages," Human Reproduction, vol. 4, no. 8, pp. 83-86, 1989.

[5] C. Ranoux and M. M. Seibel, "New techniques in fertilization: intravaginal culture and microvolume straw," Journal of In Vitro Fertilization and Embryo Transfer, vol. 7, no. 1, pp. 6-8, 1990.
[6] M. A. H. M. Wiegerinck, E. Moret, P. A. van Dop, M. Wijnberg, and C. D. M. B. Beerendonk, "Intra vaginal culture (IVC), the Eindhoven experience," in Ovulation to Implantation, J. H. L. Evers and M. J. Heineman, Eds., pp. 349351, BV: Elsevier Science, Amsterdam, The Netherlands, 1990.

[7] G. Freude, B. Artner, and S. Leodolter, "Intravaginal culture to facilitate IVF," Wiener Medizinische Wochenschrift, vol. 140, no. 20, pp. 498-501, 1990.

[8] A. L. Costoya, C. M. Cafatti, and A. A. Gadan, "Experience with intravaginal culture for in vitro fertilization (IVF)," Journal of In Vitro Fertilization and Embryo Transfer, vol. 8, no. 6, pp. 360-361, 1991.

[9] M. L. Taymor, C. J. Ranoux, and G. L. Gross, "Natural oocyte retrieval with intravaginal fertilization: a simplified approach to in vitro fertilization," Obstetrics and Gynecology, vol. 80, no. 5, pp. 888-891, 1992.

[10] S. Sharma and J. Hewitt, "Intravaginal culture for IVF," Bombay Hospital Journal, vol. 35, pp. 155-160, 1993.

[11] F. Batres, M. Mahadevan, M. Maris, M. Miller, and D. Moutos, "Stimulated cycle intravaginal culture fertilization in an office setting. A preliminary study," Fertility and Sterility, vol. 68, supplement 1, p. S168, 1997.

[12] L. Bonaventura, P. Ahlering, R. Morris, J. Mouchel, M. Scheiber, and J. Batzofin, "The INVOcell, a new medical device for intra vaginal fertilization and culture," Fertility and Sterility, vol. 86, no. 3, supplement 2, p. S164, 2006.

[13] E. Lucena, A. M. Saa, D. E. Navarro, C. Pulido, and A. Moran, "The new era in ART: INVO," Fertility and Sterility, vol. 94, supplement 4, p. S36, 2010.

[14] INVO Bioscience, INVO Cell Device: Instructions for Use P-008 Rev 7 (B ooklet), INVO Bioscience, Beverly, Mass, USA, 2007.

[15] J. Sirois, K. Sayasith, K. A. Brown, A. E. Stock, N. Bouchard, and M. Doré, "Cyclooxygenase-2 and its role in ovulation: a 2004 account," Human Reproduction Update, vol. 10, no. 5, pp. 373-385, 2004.

[16] W. Feichtinger and P. Kemeter, "Transvaginal sector scan sonography for needle guided transvaginal follicle aspiration and other applications in gynecologic routine and research," Fertility and Sterility, vol. 45, no. 5, pp. 722-725, 1986.

[17] L. L. Veek, "The human oocyte," in An Atlas of Human Gametes and Conceptuses: An Illustrated Reference for Assisted Reproductive Technology, L. L. Veeck, Ed., pp. 20-21, Parthenon Publishing Group, New York, NY, USA, 1999.

[18] World Health Organization and Department of Reproductive Health and Research, WHO Laboratory Manual for the Examination and Processing of Human Semen, vol. 56-58, World Health Organization, Geneva, Switzerland, 5th edition, 2010.

[19] E. Lucena, D. P. Bernal, C. Lucena, A. Rojas, A. Moran, and A. Lucena, "Successful ongoing pregnancies after vitrification of oocytes," Fertility and Sterility, vol. 85, no. 1, pp. 108-111, 2006.

[20] Centers for Disease Control and Prevention and Division of Reproductive Health, 2008 Assisted Reproductive Technology (ART) Report, 2009, http://www.cdc.gov/ART/ART2008/ .

[21] Centers for Disease Control and Prevention and Division of Reproductive Health, Assisted Reproductive Technology (ART) Report. 2009 National Summary Report, 2010, http://apps.nccd.cdc.gov/art/Apps/NationalSummaryReport .aspx .

[22] B. Bavister, "Oxygen concentration and preimplantation development," Reproductive BioMedicine Online, vol. 9, no. 5, pp. 484-486, 2004.

[23] M. Meintjes, S. J. Chantilis, J. D. Douglas et al., "A controlled randomized trial evaluating the effect of lowered incubator 
oxygen tension on live births in a predominantly blastocyst transfer program," Human Reproduction, vol. 24, no. 2, pp. 300-307, 2009.

[24] U. Waldenström, A. B. Engström, D. Hellberg, and S. Nilsson, "Low-oxygen compared with high-oxygen atmosphere in blastocyst culture, a prospective randomized study," Fertility and Sterility, vol. 91, no. 6, pp. 2461-2465, 2009.

[25] B. Kovačič, M. C. Sajko, and V. Vlaisavljević, "A prospective, randomized trial on the effect of atmospheric versus reduced oxygen concentration on the outcome of intracytoplasmic sperm injection cycles," Fertility and Sterility, vol. 94, no. 2, pp. 511-519, 2010.

[26] H. W. Yang, K. J. Hwang, H. C. Kwon, H. S. Kim, K. W. Choi, and K. S. Oh, "Detection of reactive oxygen species (ROS) and apoptosis in human fragmented embryos," Human Reproduction, vol. 13, no. 4, pp. 998-1002, 1998.

[27] H. J. Leese, "Metabolic control during preimplantation mammalian development," Human Reproduction Update, vol. 1, no. 1, pp. 63-72, 1995.

[28] B. Fischer and B. D. Bavister, "Oxygen tension in the oviduct and uterus of rhesus monkeys, hamsters and rabbits," Journal of Reproduction and Fertility, vol. 99, no. 2, pp. 673-679, 1993.

[29] J. E. Swain, "Optimizing the culture environment in the IVF laboratory: impact of $\mathrm{pH}$ and buffer capacity on gamete and embryo quality," Reproductive BioMedicine Online, vol. 21, no. 1, pp. 6-16, 2010.

[30] J. Q. Zhang, X. L. Li, Y. Peng, X. Guo, B. C. Heng, and G. Q. Tong, "Reduction in exposure of human embryos outside the incubator enhances embryo quality and blastulation rate," Reproductive BioMedicine Online, vol. 20, no. 4, pp. 510-515, 2010.

[31] D. K. Gardner, M. Lane, I. Calderon, and J. Leeton, "Environment of the preimplantation human embryo in vivo: metabolite analysis of oviduct and uterine fluids and metabolism of cumulus cells," Fertility and Sterility, vol. 65, no. 2, pp. 349353, 1996.

[32] V. L. Peddie and E. van Teijlingen, "Qualitative research in fertility and reproduction: does it have any value?" Human Fertility, vol. 8, no. 4, pp. 263-267, 2005.

[33] T. Jain, B. L. Harlow, and M. D. Hornstein, "Insurance coverage and outcomes of in vitro fertilization," New England Journal of Medicine, vol. 347, no. 9, pp. 661-666, 2002.

[34] O. Hovatta and I. Cooke, "Cost-effective approaches to in vitro fertilization: means to improve access," International Journal of Gynecology and Obstetrics, vol. 94, no. 3, pp. 287-291, 2006. 


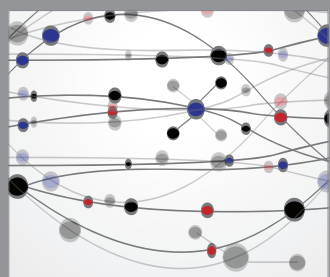

The Scientific World Journal
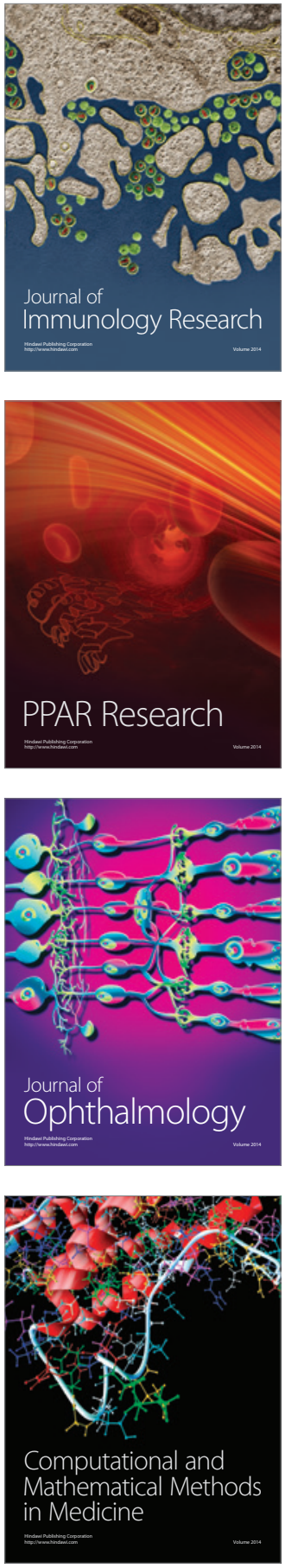

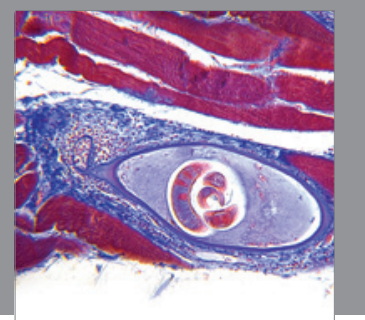

Gastroenterology

Research and Practice
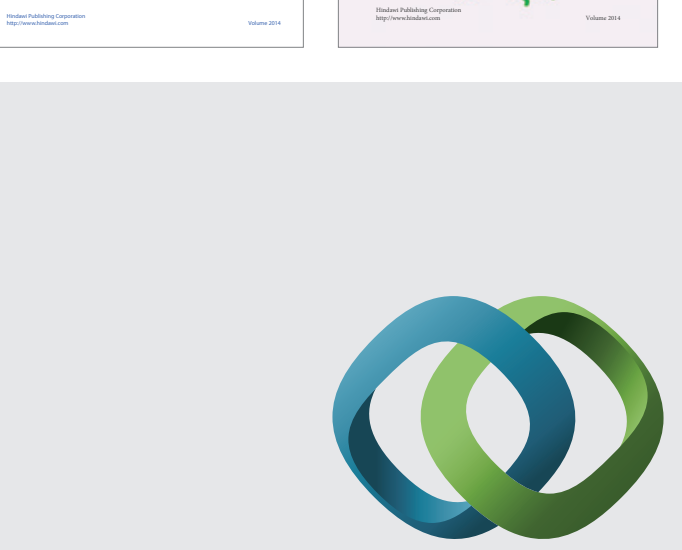

\section{Hindawi}

Submit your manuscripts at

http://www.hindawi.com
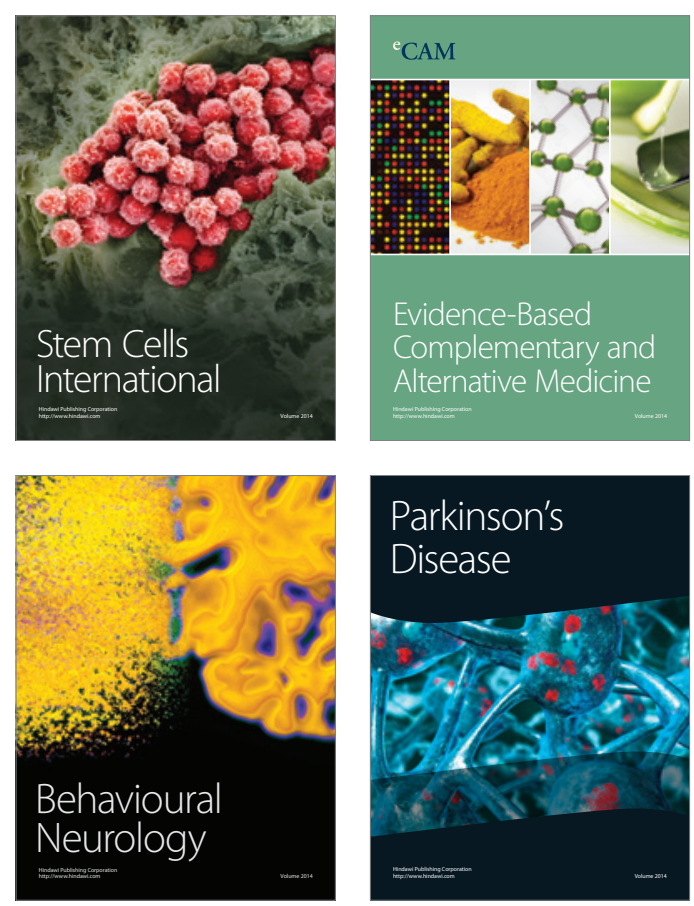

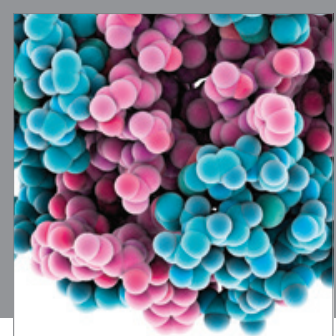

Journal of
Diabetes Research

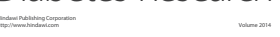

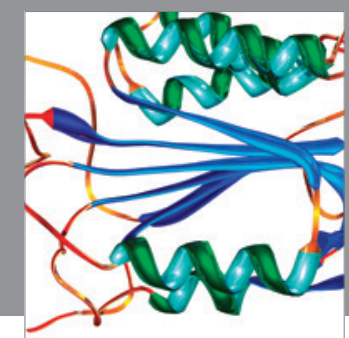

Disease Markers
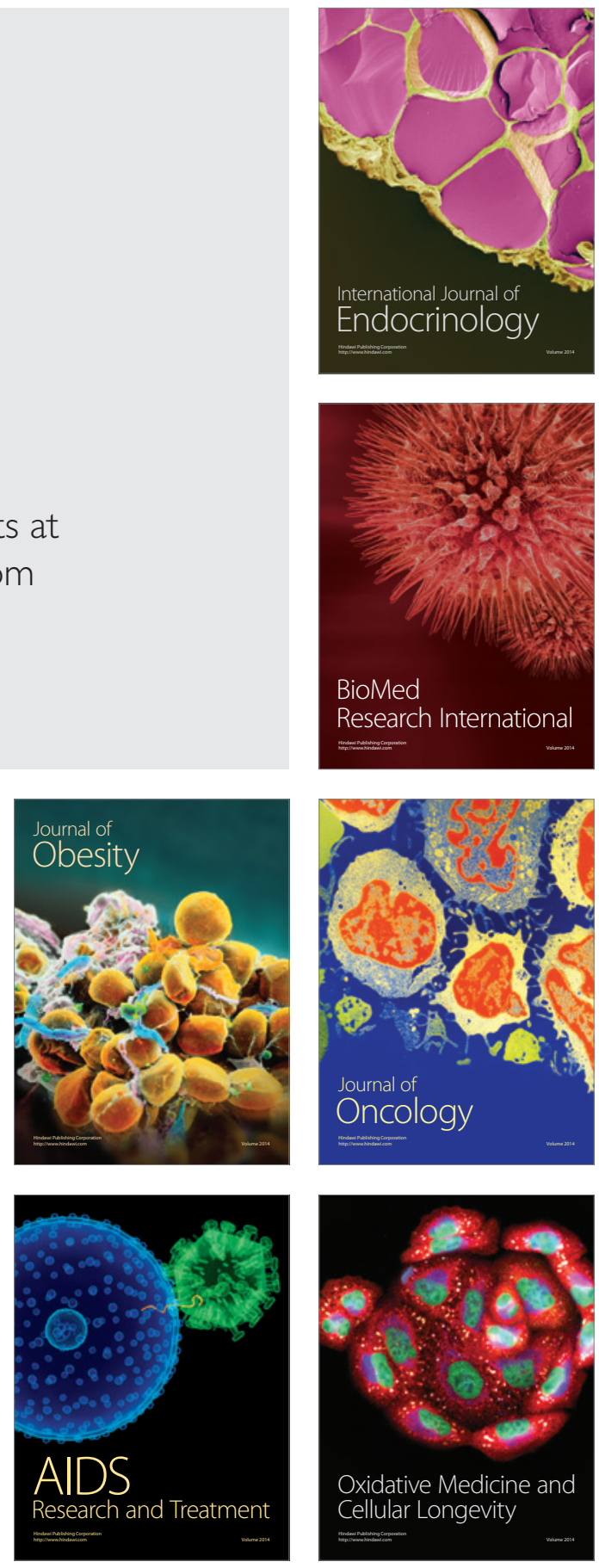